\title{
Prompt photon pair production in association with top-antitop pairs. An important background to intermediate mass Higgs detection th
}

\author{
Alessandro Ballestrero a and Ezio Maina ${ }^{\text {a,b }}$ \\ a INFN, Sezione di Torino, I-10125 Turin, Italy \\ b Dipartimento di Fisica Teorica, Università di Torino, I-10125 Turin, Italy
}

Received 26 July 1991

\begin{abstract}
The reaction $\mathrm{pp} \rightarrow \mathrm{t} \bar{\gamma} \gamma$ is studied for $80 \leqslant M_{\gamma} \leqslant 140 \mathrm{GeV}$, as a possible background to the detection of an intermediate mass standard model Higgs in the rare $\ell v r \gamma$ final state. If the top is not too heavy the prompt photon production, integrated over a window of $6 \mathrm{GeV}$ in $M_{\mathrm{r}}$ around the Higgs mass, can be larger than the production of photon pairs from Higgs decay. Standard isolation cuts can effectively dispose of this background for $m_{1} \geqslant 150 \mathrm{GeV}$. For $m_{\mathrm{t}} \sim 100 \mathrm{GeV}$ approximately the same number of background and signal events pass the cuts.
\end{abstract}

The Higgs mass range between about $80 \mathrm{GeV}$, the upper reach of LEP-200, and $140 \mathrm{GeV}$, where the Higgs boson can be detected through the gold-plated four-lepton mode, will be the most difficult to explore $[1,2]$. At hadron colliders the dominant decay mode $\mathrm{H} \rightarrow \mathrm{b} \bar{b}$ is swamped by QCD background. The $\mathrm{H} \rightarrow \gamma \gamma$ mode seems to offer the best chance because the background in the mass region of interest has been shown not to be overwhelming. Detecting $\mathrm{H} \rightarrow \gamma \gamma$ inclusively will be possible only with extremely high photon-photon mass resolution, about $1 \mathrm{GeV}$, in order to isolate the narrow Higgs enhancement over a large $\mathrm{q} \overline{\mathrm{q}}, \mathrm{gg} \rightarrow \gamma \gamma$ continuum, and with very good $\gamma$-jet discriminating power, $R_{\gamma \mathrm{j}} \simeq 10^{-4}$, necessary to eliminate background events coming from highly energetic neutral pions in low multiplicity jets. These two requirements are not easily accomplished in a general purpose detector.

Higgs bosons are naturally produced in association with heavy particles like W's and top quarks. Tagging on energetic leptons coming from the heavy partner decay, and requiring isolated and energetic photons,

\footnotetext{
* Work supported in part by Ministero dell'Università e della Ricerca Scientifica.
}

can severely reduce the background with only a modest loss in signal.

Recently the sequence $\mathrm{pp} \rightarrow \mathrm{W}^{*} \rightarrow \mathrm{WH} \rightarrow \ell \mathrm{v} \gamma \gamma, \ell=\mathrm{e}$ or $\mu$, has been examined in detail $[3,4]$, including background. These studies have focused on background processes involving light quarks and have shown that with realistic resolution in $M_{\gamma \gamma}(\Delta M \simeq \pm 3$ $\mathrm{GeV}$ ) and $\gamma$-jet discriminating power comparable to what is presently obtained by the CDF Collaboration $\left[R_{\gamma j} \simeq(3-5) \times 10^{-4}\right]$ they can be kept at a very low level with a combination of standard high- $p_{\mathrm{T}}$ and isolation cuts.

It was subsequently pointed out that, since $m_{\mathrm{t}}>m_{\mathrm{W}}+m_{\mathrm{b}}$, the associated production of a Higgs with a $\overline{\mathrm{t}} \overline{\mathrm{t}}$ pair, $\mathrm{pp} \rightarrow \mathrm{t} \overline{\mathrm{t}} \mathrm{H}$, followed by $\mathrm{t} \overline{\mathrm{t}} \rightarrow \mathrm{WbX}$ and $\mathrm{W} \rightarrow \ell v$ gives a contribution to the final $\ell v \gamma \gamma$ which is actually larger, by a factor of 5-6 at the SSC and by a factor of 2-3 at LHC, than the yield from the $\mathrm{W}^{*} \rightarrow \mathrm{WH}$ subprocess, both because of a higher production cross section and because of an increased probability of producing a lepton. This could allow detection of the Higgs at the nominal SSC yearly integrated luminosity $L=10 \mathrm{fb}^{-1}$ over the whole intermediate mass range [5,6]. At the lower energy of LHC only a luminosity $L=100 \mathrm{fb}^{-1}$, about ten times 
higher than the design one, can provide enough events for clear detection.

In this paper we study the previously neglected background coming from the production of $t \bar{t}$ pairs in association with two prompt photons. We compare the differential cross section $\mathrm{d} \sigma / \mathrm{d} M_{\gamma \gamma}(\mathrm{pp} \rightarrow$ $\overline{\mathrm{t}} \gamma \gamma)$ with the cross section $\sigma(\mathrm{pp} \rightarrow \mathrm{t} \overline{\mathrm{t}} \mathrm{H})$. Since $\mathrm{d} \sigma /$ $\mathrm{d} M_{\gamma \gamma}$ for fixed $m_{\mathrm{t}}$ is slowly varying over a few $\mathrm{GeV}$ interval in $M_{\gamma \gamma}, \Delta M \times \mathrm{d} \sigma / \mathrm{d} M_{\gamma \gamma}$ is the relevant quantity in order to assess the detectability of the intermediate mass Higgs. At Supercollider energies the contribution from $\mathrm{gg}$ collisions is much larger than the one from $q \bar{q}$ and we neglect the latter. While the cross section $\mathrm{gg} \rightarrow \mathrm{q} \overline{\mathrm{q}} \gamma \gamma$ is obviously smaller for heavy quarks than for light ones, energetic leptons are naturally produced in a large fraction of the events and the correlation between the direction of flight of the quarks and the direction of flight of the photons is much weaker in the case of the top, making isolation cuts less effective. The relatively small number of events coming from virtual W's has been neglected.

We have computed the matrix element for both

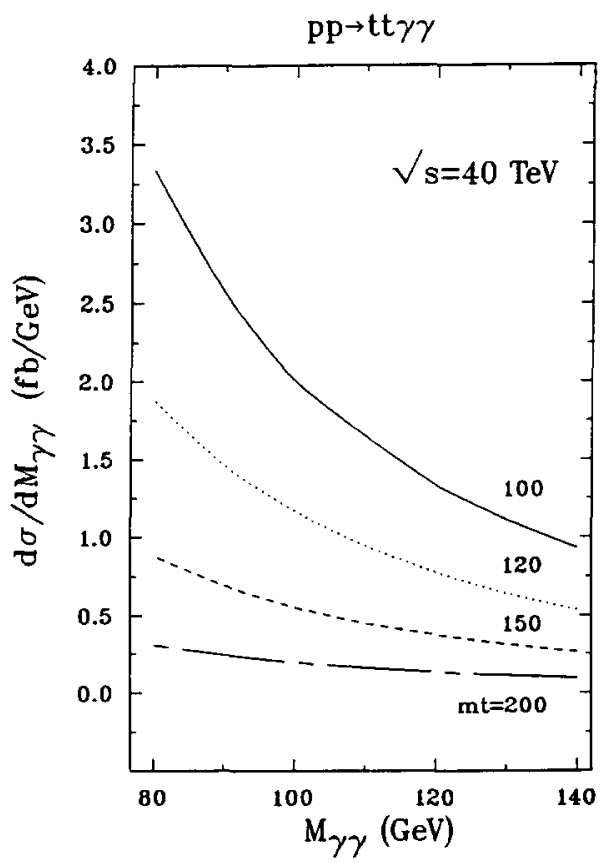

Fig. 1. $\mathrm{d} \sigma / \mathrm{d} M_{\gamma \gamma}(\mathrm{pp} \rightarrow \mathrm{t} \bar{\gamma} \gamma)(\mathrm{fb} / \mathrm{GeV})$ at $\sqrt{s}=40 \mathrm{TeV}$ for $m_{1}=$ $100,120,150,200 \mathrm{GeV}$ (continuous, dotted, dashed and chaindashed curve, respectively). processes at the amplitude level following the method of ref. [7]. In all of the calculations the structure function set HMRSB has been used [8]. The strong coupling constant has consistently been evaluated at a scale equal to the $\bar{t} \bar{t} \mathrm{H}, \mathfrak{t} \bar{t} \gamma \gamma$ subprocess invariant mass. We have used the one-loop expression for $\alpha_{\mathrm{s}}$ with $A=150 \mathrm{MeV}$ and five active flavours. Changing the scale and/or structure function choice should not affect our absolute predictions by more than a factor of two, and should leave the production cross section ratio essentially unchanged. The ratio should also be quite insensitive to QCD corrections. In figs. 1 and 2 we present $\mathrm{d} \sigma / \mathrm{d} M_{\gamma \gamma}(\mathrm{pp} \rightarrow \mathrm{tt} \gamma \gamma)$ at SSC and at LHC respectively. As expected, the prompt photon differential cross section is quite sensitive to the top mass and to $M_{\gamma \gamma}$. At SSC the event rates expected for $m_{\mathrm{t}}$ equal to 100,150 and $200 \mathrm{GeV}$ are approximately in the ratio $9: 3: 1$ for all Higgs masses. On the contrary, the $\overline{\mathrm{t} t} \mathrm{H} \rightarrow \mathrm{t} \overline{\mathrm{t}} \gamma \gamma$ yield is a slowly increasing function of $m_{\mathrm{t}}$ and essentially independent of $m_{\mathrm{H}}$ with only a slight drop for $m_{\mathrm{H}} \sim 130-140 \mathrm{GeV}$.

We have studied the effects of the cuts suggested in refs. $[4,5]$ :

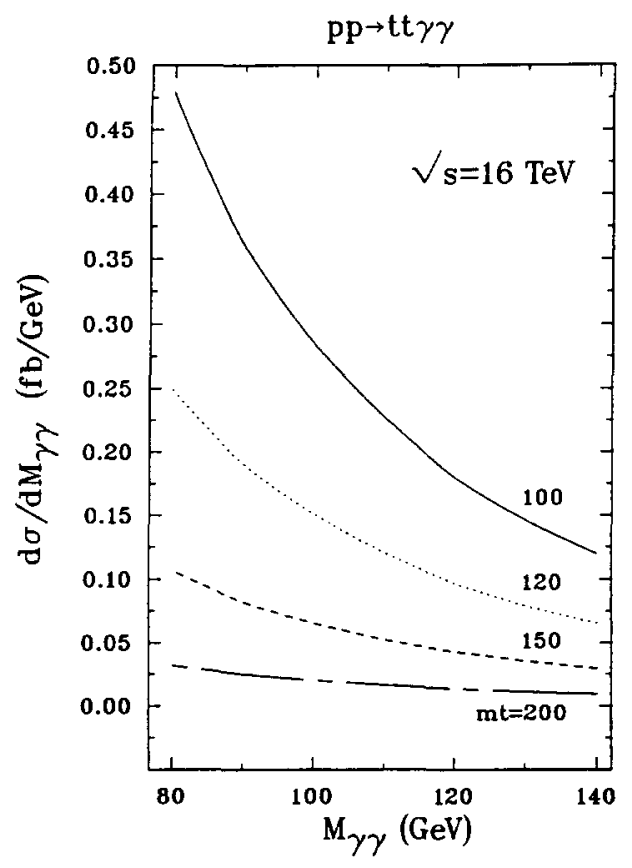

Fig. 2. $\mathrm{d} \sigma / \mathrm{d} M_{\gamma \gamma}(\mathrm{pp} \rightarrow \mathrm{t} \overline{\mathrm{t}} \gamma)(\mathrm{fb} / \mathrm{GeV})$ at $\sqrt{s}=16 \mathrm{TeV}$ for $m_{\mathrm{t}}=100$, $120,150,200 \mathrm{GeV}$ (continuous, dotted, dashed and chain-dashed curve, respectively). 


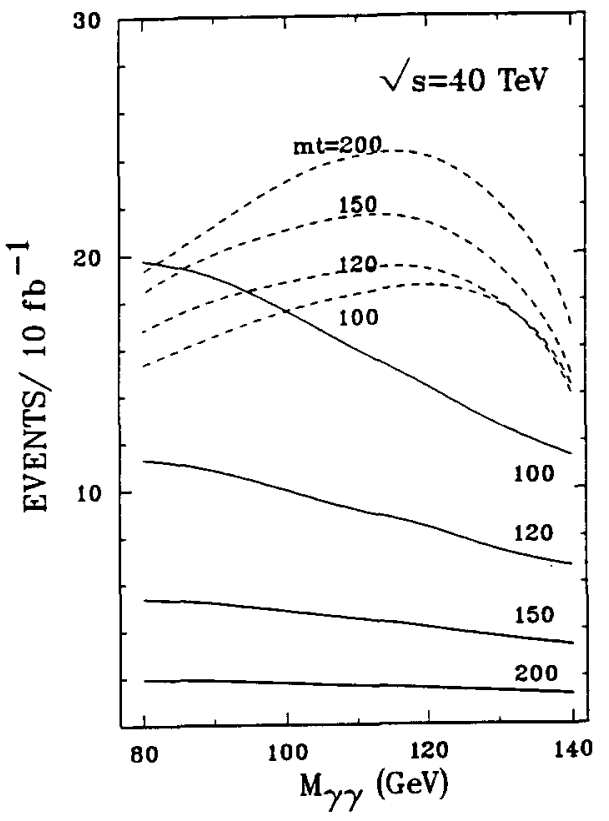

Fig. 3. Expected number of tit $\gamma \gamma$ events at SSC, in one year at the design luminosity, with the cuts described in the text, from prompt photons (continuous curves) and from $\mathbf{t} \bar{H}$ (dashed) in a window $\Delta M=6 \mathrm{GeV}$ centered around the Higgs mass. Results are presented for $m_{1}=100,120,150,200 \mathrm{GeV}$.

$$
\begin{aligned}
& \Delta R\left(\gamma_{1}, \gamma_{2}\right)>0.4, \quad \Delta R(\ell, \gamma)>0.4, \\
& p_{\mathrm{T}}(\ell, \gamma)>20 \mathrm{GeV}, \quad|\eta(\ell, \gamma)|<2.5, \\
& \left|p_{\mathrm{T}}\left(\gamma_{1}\right)+p_{\mathrm{T}}\left(\gamma_{2}\right)\right|>20 \mathrm{GeV},
\end{aligned}
$$

on both signal and background. Our results are presented in figs. 3 and 4 where we compare, for the two Supercolliders, the expected number of prompt photon $\mathrm{t} \overline{\mathrm{t}} \gamma \gamma$ events in a window of $6 \mathrm{GeV}$ centered around the Higgs mass, with the number of events from $\mathrm{pp} \rightarrow \mathrm{t} \overline{\mathrm{t}} \mathrm{H} \rightarrow \mathrm{t} \overline{\mathrm{t}} \gamma$, assuming $L=10 \mathrm{fb}^{-1}$ at SSC and $L=100 \mathrm{fb}^{-1}$ at LHC. The branching ratio $\mathrm{BR}(\mathrm{H} \rightarrow \gamma \gamma)$ has been taken from ref. [5] and includes QCD corrections to the $\mathrm{H} \rightarrow \mathrm{b} \overline{\mathrm{b}}$ width which increase the lowest order result by a factor of two. QCD corrections to the $\mathbf{H} \rightarrow \gamma \gamma$ width are neglected but have been shown in ref. [9] to be very small. The branching ratio $\mathrm{BR}(\mathrm{t} \overline{\mathrm{t}} \rightarrow \ell \vee \mathrm{X})=0.4, \ell=\mathrm{e}$ or $\mu$, is included in our results. The mentioned cuts reduce the prompt photon cross section by $75 \%$ for $M_{r \gamma}=80 \mathrm{GeV}$

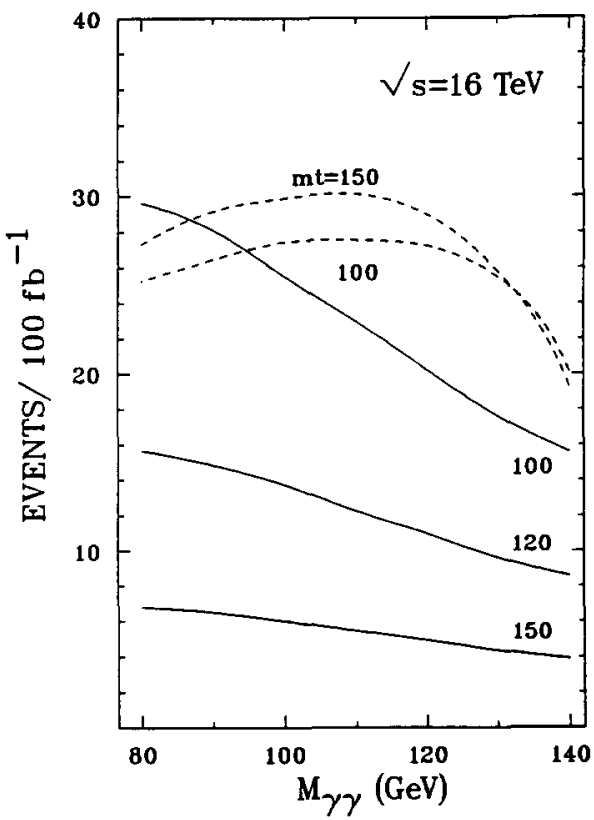

Fig. 4. Expected number of $\bar{t} \bar{\gamma} \gamma$ events at LHC, in one year at $L=100 \mathrm{fb}^{-1}$, ten times the design luminosity, with the cuts described in the text, from prompt photons (continuous curves) and from $\mathrm{tt}^{\overline{\mathrm{H}}} \mathrm{H}$ (dashed) in a window $\Delta M=6 \mathrm{GeV}$ centered around the Higgs mass. Results are presented for $m_{1}=100,150 \mathrm{GeV}$. For $m_{1}=120 \mathrm{GeV}$ we give only the prompt photon result.

and by about $50 \%$ for $M_{\gamma \gamma}=140 \mathrm{GeV}$ as can be inferred from a comparison of figs. 1 and 3 . The corresponding figures for the Higgs signal are typically $50 \%$ and $30 \%$.

At SSC the background is comparable to the Higgs signal over the whole mass range for $m_{\mathrm{t}}=100 \mathrm{GeV}$, and it is more than half of it for $m_{\mathrm{t}}=120 \mathrm{GeV}$. Therefore, while a photon-photon mass resolution of \pm 3 $\mathrm{GeV}$ seems adequate for $m_{\mathrm{t}} \geqslant 150 \mathrm{GeV}$, it is not obvious that it will be sufficient for lower top masses. The low $m_{\mathrm{t}}$ region is the most troublesome for several reason. First of all, after cuts, there are only about 15-18 lv $\gamma \gamma$ signal events. Furthermore, the top being relatively light, the tagged leptons will be on average softer than they would be for a heavier top mass. As a consequence the contribution from $p p \rightarrow b \bar{b} \gamma \gamma$ with one $\mathrm{b}$ producing a high- $p_{\mathrm{T}}$ charged lepton could be non-negligible, complicating things even further. Since the number of expected events is uncomfortably small it is particularly important to know what 
fraction of the signal will be lost to the need for a clear separation between tagged photons and leptons and the debris from the decay of the two tops and from the underlying hadronic activity. A full simulation, including jets and photons from bottom and top decay, is clearly required. Provided the required higher luminosity can be achieved, and be dealt with experimentally, everything we have said for SSC can be applied to LHC with minor modifications. There, adding the events from $W^{*} \rightarrow W H$ to the ones from $t \bar{t} H$ can somewhat improve the signal to background ratio.

In conclusion we have examined the reaction $\mathrm{pp} \rightarrow \mathrm{t} \overline{\mathrm{t}} \gamma \gamma$ and have shown it to give an important contribution to the $\ell v \gamma \gamma$ final state. The relevance of this background to Higgs detection in the intermediate mass range depends crucially on $m_{\mathrm{t}}$. If $m_{\mathrm{t}} \geqslant 150 \mathrm{GeV}$ the small number of prompt photon events does not pose a problem. For lower top masses, with a photon-photon mass resolution of $\pm 3 \mathrm{GeV}$, background and signal are comparable over the whole mass range. The standard cuts used to reduce the light quark background do not seem to be very effective in this case. Higher luminosity or better photon-photon mass resolution might be required in order to guarantee an overlap between the Higgs mass range which can be covered in LEP-200 and the mass range studied at Supercolliders.

Note added in proof. After we completed our paper we became aware of the existence of ref. [10] where the process $\mathrm{gg} \rightarrow \mathrm{t} \overline{\mathrm{t}} \gamma \gamma$ is also computed. The matrix elements squared computed by the two groups have been compared and found in complete agreement. To our knowledge our calculation of $g g \rightarrow \mathfrak{t} \mathrm{H}$ is to date the only independent confirmation of the results first obtained in ref. [11].

\section{References}

[1] S. Jensen, ed., Proc. 1988 Snowmass Workshop (World Scientific, Singapore, 1989);

J.F. Gunion et al., Overview and recent progress in Higgs boson physics at the SSC, in: Proc. 1990 Snowmass Workshop, preprint PRINT-91-0010 (1990), to appear.

[2] G. Jarlskog and D. Rein, eds., Proc. ECFA Large Hadron Collider Workshop (Aachen, 1990).

[3] R. Kleiss, Z. Kunszt and W.J. Stirling, Phys. Lett. B 253 (1991) 269

[4] M.L. Mangano, SDC Collaboration Note SSC-SDC-9000113.

[5] J.F. Gunion, Phys. Lett. B 261 ( 1991 ) 510.

[6] W.J. Marciano and F.E. Paige, preprint BNL-45805.

[7] K. Hagiwara and D. Zeppenfeld, Nucl. Phys. B 274 (1986) 1.

[8] P.N. Harriman, A.D. Martin, W.J. Stirling and R.G. Roberts, Phys. Rev. D 42 (1990) 798.

[9] A. Djouadi, M. Spira, J.J. van der Bij and P.M. Zerwas, Phys. Lett. B 257 (1991) 187.

[10] Z. Kunszt, Z. Trócsányi and W.J. Stirling, Clear signal of intermediate Higgs boson production at $\mathrm{LHC}$ and SSC, preprint ETH-TH/91-17 and DTP/91/40 (June 1991).

[11] Z. Kunzt, Nucl. Phys. B 247 (1984) 339. 